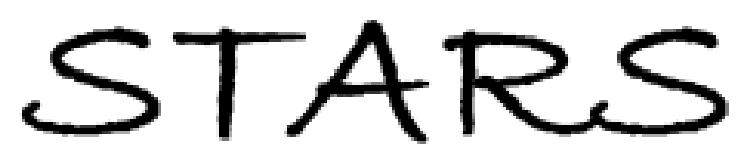

University of Central Florida

STARS

$1-1-2011$

\title{
A large Kerr constant polymer-stabilized blue phase liquid crystal
}

Linghui Rao

University of Central Florida

Jin Yan

University of Central Florida

Shin-Tson Wu

University of Central Florida

Shin-ichi Yamamoto

Yashuhiro Haseba

Find similar works at: https://stars.library.ucf.edu/facultybib2010 University of Central Florida Libraries http://library.ucf.edu

This Article is brought to you for free and open access by the Faculty Bibliography at STARS. It has been accepted for inclusion in Faculty Bibliography 2010 s by an authorized administrator of STARS. For more information, please contact STARS@ucf.edu.

\section{Recommended Citation}

Rao, Linghui; Yan, Jin; Wu, Shin-Tson; Yamamoto, Shin-ichi; and Haseba, Yashuhiro, "A large Kerr constant polymer-stabilized blue phase liquid crystal" (2011). Faculty Bibliography 2010s. 1798.

https://stars.library.ucf.edu/facultybib2010/1798

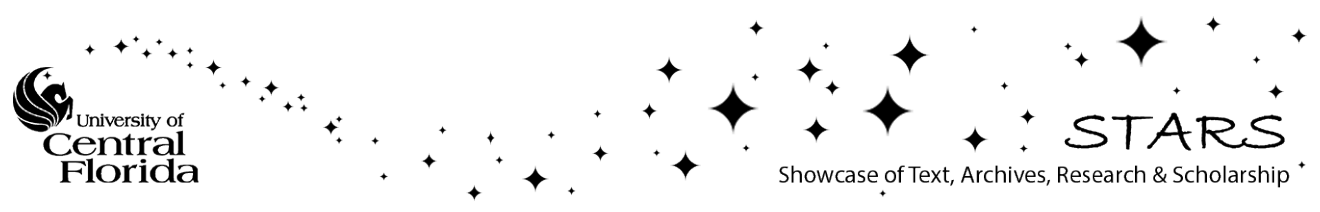




\section{A large Kerr constant polymer-stabilized blue phase liquid crystal}

Cite as: Appl. Phys. Lett. 98, 081109 (2011); https://doi.org/10.1063/1.3559614

Submitted: 26 January 2011 . Accepted: 04 February 2011 . Published Online: 24 February 2011

Linghui Rao, Jin Yan, Shin-Tson Wu, Shin-ichi Yamamoto, and Yasuhiro Haseba

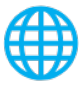

\section{ARTICLES YOU MAY BE INTERESTED IN}

Electro-optics of polymer-stabilized blue phase liquid crystal displays

Applied Physics Letters 94, 101104 (2009); https://doi.org/10.1063/1.3097355

Extended Kerr effect of polymer-stabilized blue-phase liquid crystals

Applied Physics Letters 96, 071105 (2010); https://doi.org/10.1063/1.3318288

Low voltage blue-phase liquid crystal displays

Applied Physics Letters 95, 231101 (2009); https://doi.org/10.1063/1.3271771

\section{Applied Physics Reviews} Now accepting original research. 


\title{
A large Kerr constant polymer-stabilized blue phase liquid crystal
}

\author{
Linghui Rao, ${ }^{1}$ Jin Yan, ${ }^{1}$ Shin-Tson Wu, ${ }^{1, a)}$ Shin-ichi Yamamoto, ${ }^{2}$ and Yasuhiro Haseba ${ }^{2}$ \\ ${ }^{1}$ College of Optics and Photonics, University of Central Florida, Orlando, Florida 32816, USA \\ ${ }^{2}$ Chisso Petrochemical Corporation, Ichihara, Chiba 290-8551, Japan
}

(Received 26 January 2011; accepted 4 February 2011; published online 24 February 2011)

\begin{abstract}
A polymer-stabilized blue phase liquid crystal (BPLC) composite with a large Kerr constant ( $K$ $\sim 13.7 \mathrm{~nm} / \mathrm{V}^{2}$ ) is developed and its electro-optic properties characterized. In addition to the reduced operating voltage, this BPLC also exhibits a fast response time $(\sim 1 \mathrm{~ms})$, high contrast ratio $(>1000: 1)$, and relatively small hysteresis $(<6 \%)$. It will undoubtedly accelerate the emergence of BPLC for next-generation display and photonic devices. (C) 2011 American Institute of Physics.
\end{abstract} [doi:10.1063/1.3559614]

Polymer-stabilized blue phase liquid crystal (BPLC) (Refs. 1-3) is emerging as next-generation display technology because it exhibits some very attractive features such as: (1) submillisecond gray-to-gray response time, ${ }^{4}$ (2) no need for surface alignment layer, (3) optically isotropic dark state for wide and symmetric viewing angle, and (4) cell gap insensitivity if an in-plane-switching (IPS) cell is employed. ${ }^{5}$ However, high operating voltage, low contrast ratio due to residual birefringence, and hysteresis still hinder its widespread applications. ${ }^{6,7}$ To reduce operating voltage, two approaches are commonly practiced: to develop BPLC materials with a large Kerr constant $(K)$, and to use narrow electrode gap or protrusion electrode for generating strong and deep-penetrating electric fields. ${ }^{8-11}$ Most of the BPLC materials developed so far have a $K$ value ranging from $0.4-4 \mathrm{~nm} / \mathrm{V}^{2}{ }^{12}$ The corresponding operating voltage is over $100 \mathrm{~V}$, which is too high to be driven by amorphoussilicon thin-film transistors (a-Si TFTs). There is an urgent need to develop BPLC materials with larger Kerr constant in order to lower the operating voltage.

For a given pitch length, the Kerr constant of a BPLC is primarily determined by the product of birefringence $(\Delta n)$ and dielectric anisotropy $(\Delta \varepsilon)$ of the host nematic LC. ${ }^{13}$ Another critical LC requirement for TFT addressing is high resistivity in order to avoid image flickering. To achieve high resistivity, fluorinated compounds are commonly used. The UV-stable wide nematic range fluoro mixtures usually have $\Delta n<0.25$, depending on the molecular conjugation length. Therefore, an alternative approach is to increase $\Delta \varepsilon$.

In this paper, we report a BPLC material, designated as JC-BP01M, with $\Delta n \sim 0.17$ and $\Delta \varepsilon \sim 94$. Its corresponding Kerr constant is $K \sim 13.7 \mathrm{~nm} / \mathrm{V}^{2}$ at $\lambda=633 \mathrm{~nm}$, which is $3 \times-10 \times$ higher than that reported previously. In addition to reduced on-state voltage $(\sim 48 \mathrm{~V}), \mathrm{JC}-\mathrm{BP} 01 \mathrm{M}$ also exhibits a fast response time $(\sim 1 \mathrm{~ms})$, high contrast ratio $(>1000: 1)$, and relatively low hysteresis $(<6 \%)$.

Macroscopically, BPLC is an isotropic Kerr medium when there is no external electric field $(E)$ present. As $E$ increases, the BPLC becomes anisotropic along the electric field direction. The induced birefringence is related to $E$, wavelength $\lambda$, and Kerr constant $K$ as:

${ }^{a)}$ Electronic mail: swu@ mail.ucf.edu.

$$
\Delta n_{\text {ind }}=\lambda K E^{2} .
$$

However, Eq. (1) is valid only when the electric field is weak. As $E$ keeps increasing, the induced birefringence will gradually saturate as described by the extended Kerr effect model $^{14}$

$$
\Delta n_{\text {ind }}=\Delta n_{s}\left\{1-\exp \left[-\left(E / E_{s}\right)^{2}\right]\right\},
$$

where $\Delta n_{s}$ denotes the saturated induced birefringence and $E_{s}$ the saturation electric field. In the weak field region $(E$ $\ll E_{s}$ ), we can expand Eq. (2) and deduce the Kerr constant as:

$$
K \approx \Delta n_{s} /\left(\lambda E_{s}^{2}\right) .
$$

From Eq. (3), high $\Delta n_{s}$ and low $E_{s}$ play equally important roles for enhancing Kerr constant. Roughly speaking, $\Delta n_{s}$ governs the optical property (e.g., phase change) while $E_{s}$ determines the electric property (operating voltage) of a BPLC material.

In our experiment, we injected JC-BP01M into an IPS cell with cell gap $7.5 \mu \mathrm{m}$, indium tin oxide electrode width $10 \mu \mathrm{m}$ and electrode gap $10 \mu \mathrm{m}$. We then heated the cell from chiral nematic phase to blue phase with the temperature slightly higher than the BP-I transition temperature. We held the temperature there for $1 \mathrm{~min}$ and then conducted UV curing (intensity $\sim 20 \mathrm{~mW} / \mathrm{cm}^{2}$ and $\lambda \sim 365 \mathrm{~nm}$ ). After polymerization, the clearing temperature of the BPLC composite

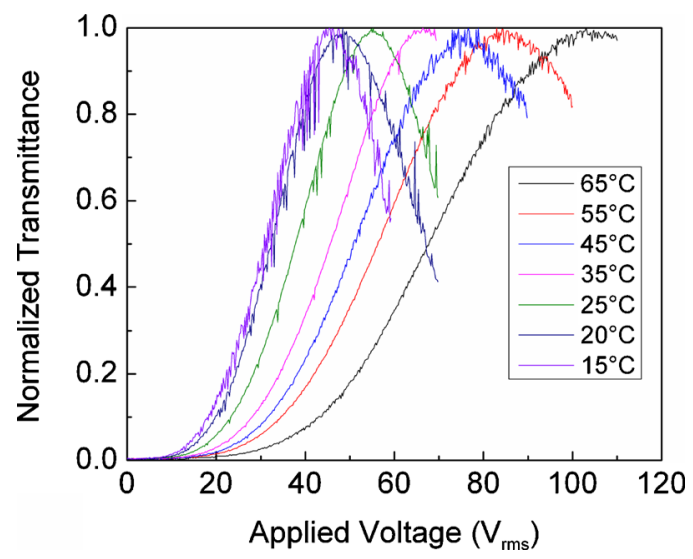

FIG. 1. (Color online) Measured VT curves of the IPS BPLC cell at elevated temperatures. Electrode width $=10 \mu \mathrm{m}$, electrode gap $=10 \mu \mathrm{m}$, and cell gap $=7.5 \mu \mathrm{m} . \lambda=633 \mathrm{~nm}$. 


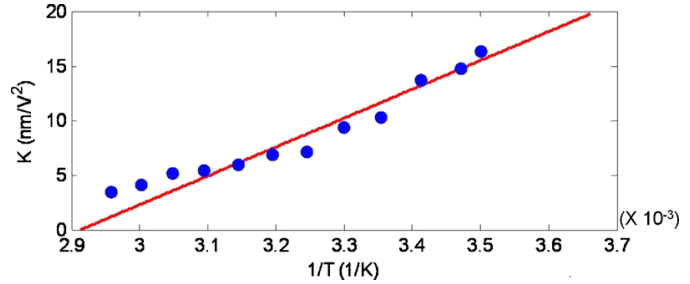

FIG. 2. (Color online) Linear fit of the Kerr constant with the reciprocal of temperature according to Eq. (4). T: Kelvin temperature. The fitting parameter is $\alpha=2.644 \times 10^{-5} \mathrm{~m} \mathrm{~K} / \mathrm{V}^{2}$.

was measured to be $T_{c} \sim 70{ }^{\circ} \mathrm{C}$. For a transmissive display, the BPLC device should have no reflection in the visible region. Thus, we designed our cholesteric pitch to be $\sim 200 \mathrm{~nm}$ so the Bragg reflection occurs at $\sim 350 \mathrm{~nm}$.

We measured the voltage-dependent transmittance (VT) of our IPS cell with a He-Ne laser $(\lambda=633 \mathrm{~nm})$. Figure 1 shows the normalized VT curves from 15 to $65{ }^{\circ} \mathrm{C}$. As the temperature increases, the on-state voltage $\left(V_{\text {on }}\right)$ increases. We fitted the VT curves shown in Fig. 1 with the extended Kerr effect model [Eqs. (2) and (3)] at each temperature and obtained the $K$ values as plotted in Fig. 2. The temperaturedependent Kerr constant is linearly proportional to the reciprocal temperature $(1 / T)$ as: ${ }^{15}$

$$
K=\alpha \cdot\left(\frac{1}{T}-\frac{1}{T_{c}}\right)
$$

Here, $\alpha$ is the proportionality constant and $T_{c}$ is the clearing point. To fit the experimental data with Eq. (4), we used $T_{c}$ $=343.15 \mathrm{~K}\left(70{ }^{\circ} \mathrm{C}\right)$ and obtained $\alpha=2.644 \times 10^{-5} \mathrm{~m} \mathrm{~K} / \mathrm{V}^{2}$.

From Fig. 2, JC-BP01M has a Kerr constant $K$ $\sim 13.7 \mathrm{~nm} / \mathrm{V}^{2}$ at $20{ }^{\circ} \mathrm{C}$, which corresponds to $V_{\text {on }}$ $\sim 48 V_{\text {rms }}$ (at $\lambda=633 \mathrm{~nm}$ ) in our IPS cell. For a green wavelength at $\lambda=550 \mathrm{~nm}$, the $V_{\text {on }}$ is estimated to be $\sim 43 V_{\text {rms }}$. If we employ JC-BP01M in a cell with protruded electrodes, ${ }^{8}$ the operating voltage would drop to below 10V. This will enable BPLC to be driven by a-Si TFT which will accelerate the emergence of BPLC for display and photonics applications. ${ }^{16,17}$

By fitting the VT curves shown in Fig. 1 with extended Kerr effect, we also obtained $\Delta n_{s}$ at different temperatures. Results are plotted in Fig. 3, in which we find that $\Delta n_{s}$ decreases gradually as the temperature increases ${ }^{18}$

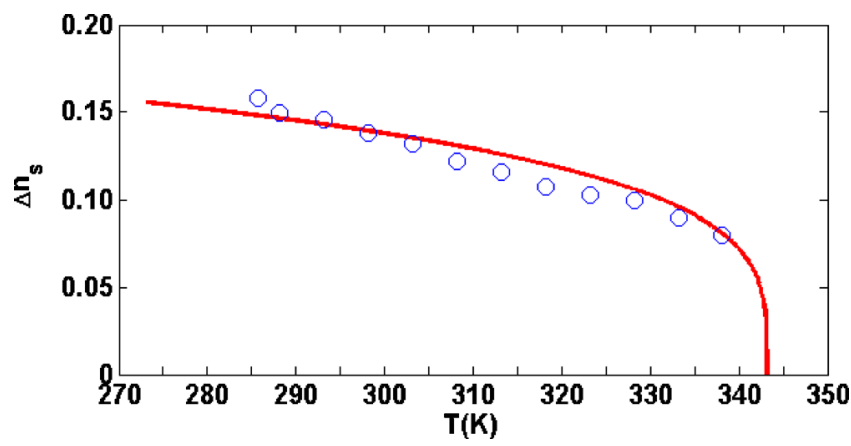

FIG. 3. (Color online) Temperature dependent saturated induced birefringence. Open circles are experimental data and solid line represents fitting using Eq. (5) with $\left(\Delta n_{s}\right)_{o}=0.232$ and $\beta=0.25$.

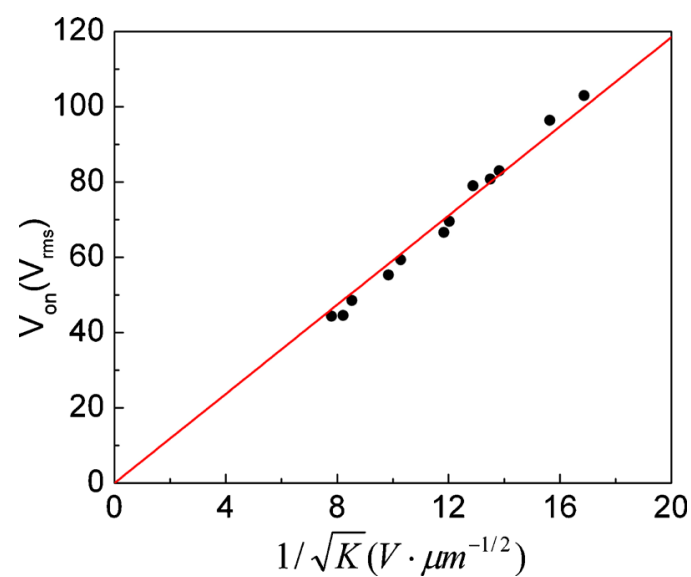

FIG. 4. (Color online) Linear fit of $V_{\text {on }}$ vs $1 / \sqrt{K}$ according to Eq. (6) with $A=5.92 \mu \mathrm{m}^{1 / 2} . \lambda=633 \mathrm{~nm}$.

$$
\Delta n_{s}=\left(\Delta n_{s}\right)_{o}\left(1-T / T_{c}\right)^{\beta},
$$

where $\left(\Delta n_{s}\right)_{o}$ is the extrapolated induced birefringence at $T=0 \mathrm{~K}$ and the exponent $\beta$ is a material constant. Equation (5) works well as long as the temperature is not too close (within $1{ }^{\circ} \mathrm{C}$ ) to the clearing point. From the fitting curve shown in Fig. 3, we find $\left(\Delta n_{s}\right)_{o}=0.232$ and $\beta=0.25$. For many nematic LC materials studied, $\beta$ is around $0.19-0.26 .^{19,20}$ A larger $\beta$ implies to a smaller order parameter.

Kerr constant affects the on-state voltage of the BPLC cell. From Fig. 1, we obtain the on-state voltage at different temperatures and then plot $V_{\text {on }}$ against $1 / \sqrt{K}$ in Fig. 4. Our previous simulation results predict the following relationship between on-state voltage and Kerr constant ${ }^{8}$

$$
V_{\text {on }}=A / \sqrt{K} \text {. }
$$

In Eq. (6), $A$ is a device parameter which is affected by the electrode configuration. The model fits well as the straight line shows in Fig. 4. From the slope, we find $A$ $=5.92 \mu \mathrm{m}^{1 / 2}$. This result agrees well with the same IPS structure but with a low- $K$ BPLC material, in which $A$ $=6.07 \mu \mathrm{m}^{1 / 2} .{ }^{15}$ From Eq. (6), both device configuration (thru $A$ ) and large- $K$ material play important roles for lowering the operating voltage.

Fast response time is one of the most attractive features of BPLC since it helps to reduce motion blur and enables color-sequential display while minimizing color breakup. We measured the decay time of our IPS cell from 12.5 to $65^{\circ} \mathrm{C}$, and results are plotted in Fig. 5. The decay time (90\%-10\% transmittance change) decreases as the temperature increases, and reaches $\sim 1 \mathrm{~ms}$ at $\sim 30^{\circ} \mathrm{C}$. We use the following equation to fit the experimental data: ${ }^{15}$

$$
\tau \approx B \cdot \frac{\exp \left(E_{a} / K_{B} T\right)}{\left(1-T / T_{c}\right)^{\beta}},
$$

where $B$ is the proportionality constant, $E_{a}$ is the activation energy of molecular rotation, and $K_{B}$ is the Boltzmann constant. While deriving Eq. (7), we have assumed the pitch length is insensitive to the temperature. This assumption is validated by our experimental observation. In our polymerstabilized BPLC cell, the reflection wavelength does not change noticeably with the temperature. This is because the BPLC lattice structure is stabilized by the polymer networks. 


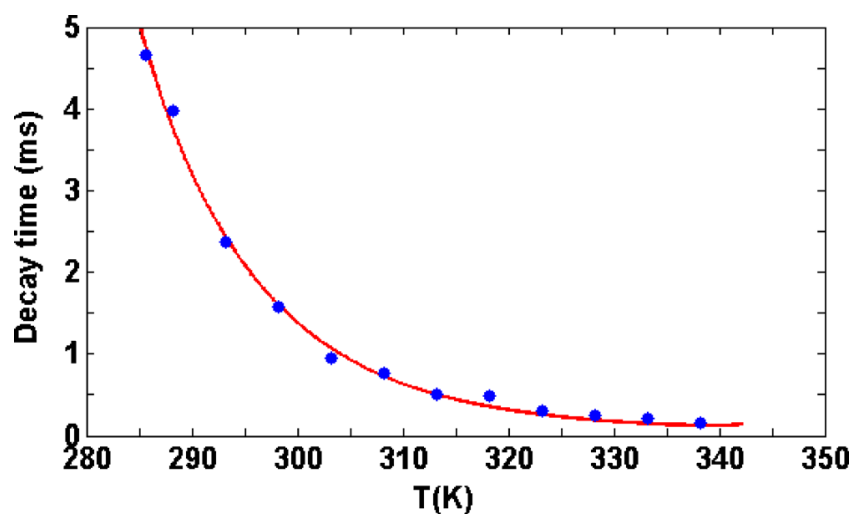

FIG. 5. (Color online) Temperature dependent decay time. Dots are experimental data and line represents fitting using Eq. (7) with $B=4.05$ $\times 10^{-12} \mathrm{~ms}$ and $E_{a}=687.75 \mathrm{meV}$.

Through fitting, we find $B=4.05 \times 10^{-12} \mathrm{~ms}$ and $E_{a}$ $=687.75 \mathrm{meV}$. The fairly large $E_{a}$ results from the increased viscosity of the huge $\Delta \varepsilon$ nematic host and chiral dopant. ${ }^{21}$ From Fig. 5, as the temperature increases by $\sim 10{ }^{\circ} \mathrm{C}$, the response time decreases by $\sim 2 \times$. This changing rate is $\sim 2$ $\times$ faster than a typical nematic because of the higher $E_{a}$ of our BPLC.

Polymer-stabilized LCs often exhibit memory effect, also known as hysteresis, which affects the accuracy of grayscale control and should be minimized. ${ }^{7}$ We have measured the VT curves of our sample for two cycles of ascending and descending voltage scans at $25{ }^{\circ} \mathrm{C}$ and $\lambda=633 \mathrm{~nm}$. Hysteresis is defined as the voltage difference at half-maximum transmittance between voltage-up and -down scans. From the experiment, we found the hysteresis of our sample is $\Delta V$ $\sim 3 V_{\text {rms }}$ and the ratio of $\Delta V / V_{\mathrm{p}}$ is $\sim 6 \%$. Moreover, the measured VT curves are quite repeatable, indicating that the residual birefringence of our sample is negligible. The measured contrast ratio exceeds 1000:1. To further suppress hysteresis, uniform UV exposure, controlling the curing temperature, or slightly increasing the operation temperature will be helpful. ${ }^{7}$

In conclusion, we have demonstrated a polymerstabilized BPLC mixture with a large Kerr constant $K$ $\sim 13.7 \mathrm{~nm} / \mathrm{V}^{2}$ at $20{ }^{\circ} \mathrm{C}$ and $\lambda=633 \mathrm{~nm}$. The corresponding on-state voltage is $\sim 48 V_{\text {rms }}$ in an IPS cell with $10 \mu \mathrm{m}$ electrode width and $10 \mu \mathrm{m}$ electrode gap. If we use this material in a protrusion electrode cell, the operating voltage would be reduced to $<10 \mathrm{~V}$. The slightly slower response time $(\sim 1 \mathrm{~ms})$ is because JC-BP01M has a relatively high viscosity due to its huge $\Delta \varepsilon$. The device contrast ratio exceeds 1000:1 and the hysteresis is $\sim 6 \%$. Potential application of this mixture for next-generation display and photonic devices is foreseeable.

The UCF group is indebted to ITRI (Taiwan) and AU Optronics for financial supports.

${ }^{1}$ H. Kikuchi, M. Yokota, Y. Hisakado, H. Yang, and T. Kajiyama, Nature Mater. 1, 64 (2002)

${ }^{2}$ Y. Haseba, H. Kikuchi, T. Nagamura, and T. Kajiyama, Adv. Mater. 17, 2311 (2005)

${ }^{3}$ Z. Ge, S. Gauza, M. Jiao, H. Xianyu, and S. T. Wu, Appl. Phys. Lett. 94, 101104 (2009).

${ }^{4}$ K. M. Chen, S. Gauza, H. Xianyu, and S. T. Wu, J. Disp. Technol. 6, 49 (2010).

${ }^{5}$ Z. Ge, L. Rao, S. Gauza, and S. T. Wu, J. Disp. Technol. 5, 250 (2009).

${ }^{6}$ H. Kikuchi, Y. Haseba, S. Yamamoto, T. Iwata, and H. Higuchi, Proceedings of the SID Symposium Digest 40, 578 (2009).

${ }^{7}$ K. M. Chen, S. Gauza, H. Xianyu, and S. T. Wu, J. Disp. Technol. 6, 318 (2010).

${ }^{8}$ L. Rao, Z. Ge, S. T. Wu, and S. H. Lee, Appl. Phys. Lett. 95, 231101 (2009).

${ }^{9}$ M. Jiao, Y. Li, and S. T. Wu, Appl. Phys. Lett. 96, 011102 (2010).

${ }^{10}$ S. Yoon, M. Kim, M. S. Kim, B. G. Kang, M. K. Kim, A. K. Srivastava, S. H. Lee, Z. Ge, L. Rao, S. Gauza, and S. T. Wu, Liq. Cryst. 37, 201 (2010).

${ }^{11}$ L. Rao, Z. Ge, and S. T. Wu, J. Disp. Technol. 6, 115 (2010).

${ }^{12}$ S. W. Choi, S. Yamamoto, Y. Haseba, H. Higuchi, and H. Kikuchi, Appl. Phys. Lett. 92, 043119 (2008).

${ }^{13}$ P. R. Gerber, Mol. Cryst. Liq. Cryst. 116, 197 (1985).

${ }^{14}$ J. Yan, H. C. Cheng, S. Gauza, Y. Li, M. Jiao, L. Rao, and S. T. Wu, Appl. Phys. Lett. 96, 071105 (2010).

${ }^{15}$ L. Rao, J. Yan, and S. T. Wu, J. Soc. Inf. Disp. 18, 954 (2010).

${ }^{16}$ M. Fridman, M. Nixon, E. Ronen, A. A. Friesem, and N. Davidson, Opt. Lett. 35, 526 (2010).

${ }^{17}$ Y. H. Lin, H. S. Chen, H. C. Lin, Y. S. Tsou, H. K. Hsu, and W. Y. Li, Appl. Phys. Lett. 96, 113505 (2010).

${ }^{18}$ S. T. Wu, Phys. Rev. A 33, 1270 (1986).

${ }^{19}$ M. Schadt and F. Muller, IEEE Trans. Electron Devices 25, 1125 (1978)

${ }^{20}$ S. T. Wu, A. M. Lackner, and U. Efron, Appl. Opt. 26, 3441 (1987).

${ }^{21}$ S. T. Wu and C. S. Wu, Phys. Rev. A 42, 2219 (1990). 